\title{
Hyperspectral spectro-imager survey for state of art concept identification
}

Weerapot Wanajaroen, Thierry Lépine, Christophe Buisset, Matthieu Castelnau, Vincent Costes, et al.

Weerapot Wanajaroen, Thierry Lépine, Christophe Buisset, Matthieu Castelnau, Vincent Costes, Suwicha Wannawichian, Saran Poshyachinda, Boonrucksar Soonthornthuma, "Hyperspectral spectro-imager survey for state of art concept identification," Proc. SPIE 11852, International Conference on Space Optics - ICSO 2020, 1185268 (11 June 2021); doi: 10.1117/12.2600232

SPIE Event: International Conference on Space Optics - ICSO 2021, 2021, Online Only 


\section{International Conference on Space Optics-ICSO 2020}

Virtual Conference

30 March-2 April 2021

Edited by Bruno Cugny, Zoran Sodnik, and Nikos Karafolas
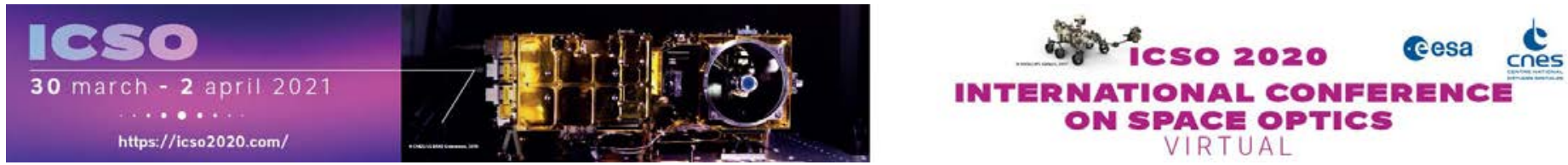

\section{Hyperspectral spectro-imager survey for state of art concept identification}

\section{Cesa isoporecestings denes}




\title{
Hyperspectral spectrometer survey for state of art concept identification
}

\author{
Weerapot Wanajaroen ${ }^{\mathrm{a}, \mathrm{b}}$, Thierry Lépine ${ }^{\mathrm{c}, \mathrm{d}}$, Christophe Buisset ${ }^{\mathrm{a}}$, Matthieu Castelnau, \\ Vincent Costes ${ }^{\mathrm{e}}$, Suwicha Wannawichian ${ }^{\mathrm{f}}$, Saran Poshyachinda ${ }^{\mathrm{a}}$, Boonrucksar Soonthornthuma ${ }^{\mathrm{a}}$ \\ ${ }^{a}$ National Astronomical Research Institute of Thailand (Public Organization), 260 Moo 4, \\ T. Donkaew, A. Maerim, Chiangmai, Thailand, 50180 \\ ${ }^{\mathrm{b}}$ Doctor of Philosophy Degree Program in Astronomy, Department of Physics and Materials \\ Science, Faculty of Science, Chiang Mai University, Chiang Mai 50200, Thailand \\ ${ }^{c}$ Institut d'Optique Graduate School , 18 rue Benoît Lauras, 42000 Saint-Etienne, France \\ ${ }^{\mathrm{d}}$ Laboratoire Hubert Curien, Université de Lyon, CNRS, 18 rue Benoît Lauras F-42000, Saint- \\ Etienne, France \\ ${ }^{\mathrm{e}}$ CNES, 18 Avenue Edouard Belin, 31401 Toulouse Cedex 9, France \\ ${ }^{\mathrm{f}}$ Department of Physics and Materials Science, Faculty of Science, Chiang Mai University, Chiang \\ Mai 50200, Thailand
}

\begin{abstract}
Over the past twenty years, a very high number of different space hyperspectral spectrometers have been designed, developed and launched. Recently, several new compact designs based on freeform optics have been proposed in the literature. We thus considered that it was interesting to make a survey of the already existing hyperspectral imagers and innovative freeform designs to identify the most promising solutions to increase the compactness and the performance.

Some surveys on hyperspectral imagers have already been done in the past. For example, the survey by Herring et al, in 1993 which proposed some hyperspectral spectrometer concepts. However, these studies are now outdated and cannot be used to identify state of art concepts. The review proposed by Kumar et al., in 2015 presented the advantages to browse the different hyperspectral imager concepts but provided only superficial technical details that cannot be used to identify the specific designs that improve both the compactness and the image and spectral qualities.

We thus decided to make a bibliographic survey to analyze the most recent designs of different space hyperspectral imagers with the objective to select the concepts that will provide the best compromise between the volume and the performance. We have categorized the different systems into four groups: prism based, Offner, Three Mirror Anastigmat (TMA) and Dyson spectrometers. We analyzed these different concepts and we identified and the advantages and the drawbacks.

We concluded that the most interesting state-of-art designs are the Freeform Offner, double-pass TMA and Dyson spectrometers. These designs present the most interesting performance and combine with a compact volume. We thus established a preliminary trade-off that summarizes the advantages and the drawback of these concepts. This trade-off analysis could be used as a starting point for any future study aiming at designing compact hyperspectral imager. Finally, we present the progress of some important and recent technological developments related to the manufacturing of freeform and convex gratings.
\end{abstract}

Keywords: hyperspectral imager, spectrometer, remote sensing, optical design.

\section{INTRODUCTION}

The objective is to design compact and high performance spectrometers. Over the past twenty years, a very high number of different space hyperspectral imagers have been designed, developed and launched. Recently, several new compact designs based on freeform optics have been proposed in the literature. We thus considered that it was mandatory to make 
a survey of the already existing hyperspectral imagers and innovative freeform designs to identify the most promising solutions to increase the compactness and the performance.

Some survey of hyperspectral imagers has already been done in the past [29], [30]. However, the study performed in 1993 by Herring et al., is now outdated and cannot be used to identified state of art concepts. The review presented by Kumar et al., in 2015 presents the advantages to browse all the different hyperspectral imager concepts but provide only superficial technical details that cannot be used to identify the specific designs that improve both the compactness and the image and spectral quality.

We thus decided to make a survey to analyze the most recent designs with the objective to select the concepts that will provide the best compromise between the volume and the performance. We have categorized the different systems in five categories: classical prism or grating based spectrometer, Offner, Three Mirror Anastigmat (TMA) and Dyson spectrometers. For each instrument, we present the design concept and the performance.

In this document, we summarize the design and the performance of a selected number of instruments. From our point of view these instruments present particularly interesting performance, overall volume or technological developments.

In Section 2, we present the design and the performance of some spectrometers based on a prism disperser. First, we describe double-pass prism-based spectrometer [1] that provides spectrum over the spectral interval [400-2500 nm] and which total length is equal to $300 \mathrm{~mm}$. Second, we summarize the design and the performance of the "compact hyperspectral prism spectrometer for sustainable land imaging" [3], [4]. This instrument has been designed to measure the spectrum of lands over several spectral bands covering the spectral interval [ $400 \mathrm{~nm}, 2.2 \mu \mathrm{m}]$.

In section 3, we first describe some Offner spectrometers. First, we describe an optical design proposed by [8] that includes one freeform mirror. This instrument is designed to cover the spectral band 240-2500 nm with a GSD varying from $4 \mathrm{~m}$ in the UV to $7.5 \mathrm{~m}$ in the SWIR and a very large FOV equal to 27 degree. Second, we present the result of a study performed by [8] focused on the possibility of improving the compactness of an Offner spectrometer by inserting freeform surfaces (including the grating surface). The proposed concept is a very compact optical system diffractionlimited over the spectral domain [200, $1900 \mathrm{~nm}]$ and over a $20 \mathrm{~mm}$ length slit.

In section 4, we present the performance obtained with Three Mirror Anastigmat (TMA) spectrometers. We mainly focus on the double-pass TMA [17] that includes only one freeform optical surface and a plane grating. This instrument presents a diffraction image quality over the spectral band $420-1000 \mathrm{~nm}$ for a quite compact volume.

In section 5, we present some important and recent technological developments related to compact imaging spectrometers. First, we summarize a paper that describes the capabilities of manufacturing freeform gratings using a 5 axis Computer Numerical Control (CNC) machine. Finally, we present the design and performance of the space instruments "SSTL spectrometer" [7] and "MicroCarb" [28].

\section{CLASSICAL PRISM AND GRATING BASED SPECTROMETER}

\subsection{Double-pass prism-based spectrometer}

This paper [1] describes the design and the performance of the double-pass prism-based spectrometer designed to provide spectrum over the spectral domain $400-2500 \mathrm{~nm}$. The F-number is equal to 4.3 , the GSD is equal to $5.7 \mathrm{~m}$ and the ground swath is equal to $2.86 \mathrm{~km}$. The design comprises one fused silica prism as a dispersive element, one retroreflecting mirror and two fused silica focal reducer lenses as shown in Figure 1. The total length of this instrument is equal to $400 \mathrm{~mm}$. The magnification is equal to 0.48 . The dispersion is equal to $8.2 \mathrm{~nm} /$ pixel. The spectrum is spread on 256 pixels.

The detector is made of one thinned HgCdTe detector that comprises 1024 x 256 pixels. This detector can operate over the specified spectral interval, from $400 \mathrm{~nm}$ to $2.5 \mu \mathrm{m}$. The HgCdTe grows on CdZnTe substrate, but the CdZnTe blocks the shorter wavelength below $850 \mathrm{~nm}$. The thinned HgCdTe is produced by removing of the CdZnTe substrate. We consider that the capability for one detector to cover the full specified spectral interval is very interesting. 


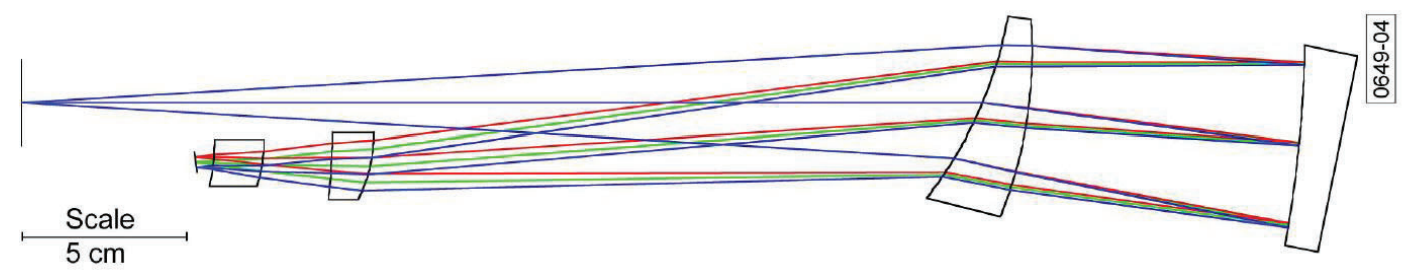

Figure 1. double-pass prism-based spectrometer optical design layout.

\subsection{Compact Hyperspectral Prism Spectrometer (CHPS)}

The Compact Hyperspectral Prism Spectrometer (CHPS) [3], [4] has been developed under the NASA's Sustainable Land Imaging-Technology (SLI-T) program. This instrument operates over the spectral domain 400-2500 nm and the design includes prisms to avoid the straylight induce by the gratings and to reduce the sensitivity of the polarization for inland and coastal water observations. This instrument provides continuous high-fidelity spectroscopic information across the solar reflected portion of the electromagnetic spectrum at the spectral sampling range from $1.3 \mathrm{~nm} / \mathrm{pixel}$ to 11 $\mathrm{nm} / \mathrm{pixel}$.

The CHPS volume is equal to $500 \times 500 \times 500 \mathrm{~mm}^{3}$ that is 30 times smaller than Landsat- 8 .. The CHPS airborne version (CHPS-AB) provides $2.5 \mathrm{~m}$ GSD with $1.41 \mathrm{~km}$ swath at an altitude of the aircraft equal to $4 \mathrm{~km}$. The CHPS space borne version will provide a GSD equal to $30 \mathrm{~m}$ at a spacecraft altitude equal to $704 \mathrm{~km}$. The CHPS-AB dispersive elements include a curved aplanatic prism in a double-pass configuration following by one spherical mirror. Two additional fused silica elements are used as field lenses. The first field lens is located next to the slit. The second field lens is placed in front of the focal plane as shown in Figure 2. The detector format is $2 \mathrm{k} \mathrm{x} 2 \mathrm{k}$ pixels with $12 \mu \mathrm{m}$ pixel size. The dispersion of the CHSP-AB varies between $2.3 \mathrm{~nm} / 2$ binning pixels to $21.3 \mathrm{~nm} / 2$ binning pixels.

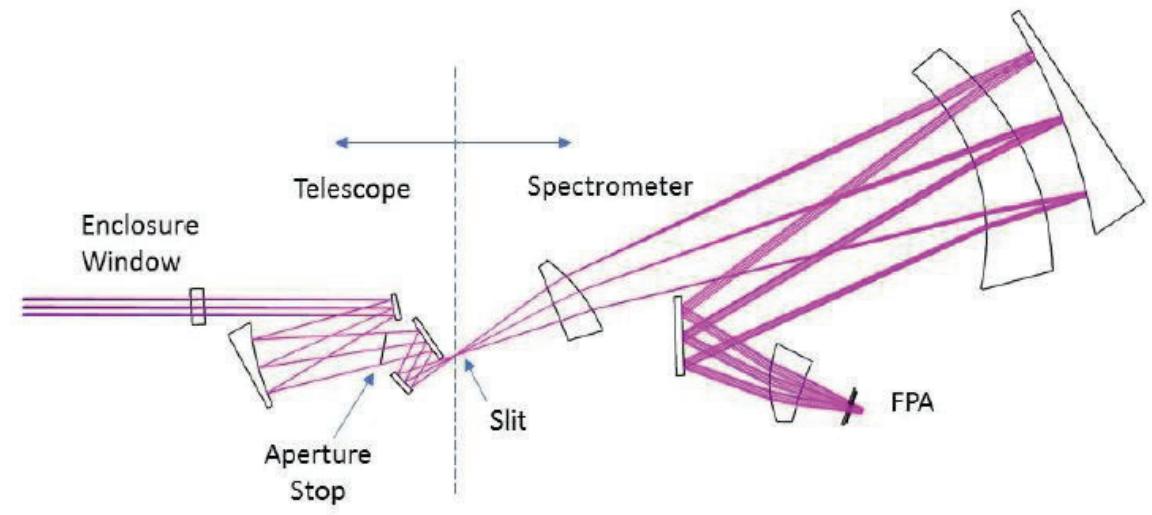

Figure 2. Optical design layout of CHPS. The front telescope is on the left side of the line. The spectrometer is on the right side of the line.

\section{OFFNER SPECTROMETER}

\subsection{Wide field freeform Offner spectrometer}

The compact spectrometer presented in [7] has been designed to provide a large field of view (27 degree) and a spectral range equal to $240-2500 \mathrm{~nm}$ thus covering the UV, VNIR and SWIR bands. The GSD is specified to be equal to $4 \mathrm{~m}$ in the VNIR and $7.5 \mathrm{~m}$ in the SWIR.

The spectrometer is an Offner that comprises one grating, one filter, three mirrors and two folding mirrors. The spectrometer layout is presented in Figure 3. The primary mirror M1 is a freeform surface, the reflective grating (M2) is manufactured on a convex spherical surface and the tertiary mirror M3 is spherical and concave. 
M3 reflects the incident beam toward the filter set that is considered as the most critical part of the instrument. This filter comprises one filter for the UV band, one filter for the VNIR band and one filter for the SWIR band. These multilayer filters are used to select spectral band incident on each detector.

This instrument includes folding mirrors located in front of the image plane to spatially separate the beam in the UV, VNIR and SWIR spectral bands. The beam corresponding each spectral band is focused on a detector. The pixel sizes are equal to $13.5 \mu \mathrm{m}$ in UV-VNIR and is equal to $25 \mu \mathrm{m}$ in the SWIR. The dispersion in the UV-VNIR (respectively in the SWIR) is close to $4 \mathrm{~nm} /$ pixel (respectively equal to $25 \mathrm{~nm} /$ pixel). The volume of the payload (telescope + spectrometer) is equal to $316 \times 160 \times 170 \mathrm{~mm}^{3}$. The flight altitude is equal to $30 \mathrm{~km}$.

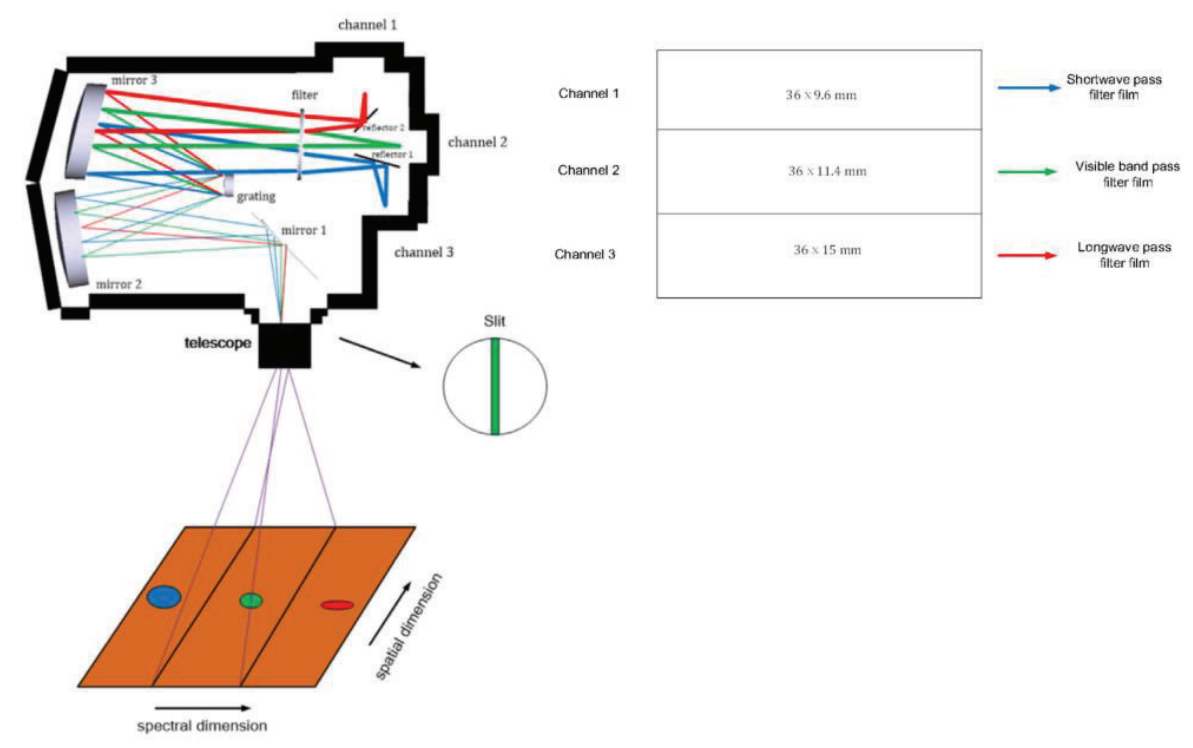

Figure 3. Wide field freeform Offner spectrometer optical design layout with three channel focal planes.

\subsection{Compact freeform Offner spectrometer}

The paper [8] presents the advantages provided by freeform optics to reduce the volume and increase the image and spectral performance of an Offner spectrometer. The spectral domain is equal to $200-1500 \mathrm{~nm}$, the beam aperture is equal to $\mathrm{f} / 3.8$ and the spectral dispersion is equal to $100 \mathrm{~nm} / \mathrm{mm}$. The grating density is equal to $150 \mathrm{line} / \mathrm{mm}$ and the FOV is defined by the slit length that is equal to $10 \mathrm{~mm}$.

The starting point is the all-spherical Offner - Chrisp spectrometer that presents a problem of astigmatism over the spectral domain [200, $500 \mathrm{~nm}$ ]. This instrument can provide an acceptable image quality over [500 nm, $1100 \mathrm{~nm}$ ] only. The volume of this spectrometer is equal to $530 \mathrm{~cm}^{3}$. The author shows that if we reduce the volume to $100 \mathrm{~cm}^{3} \mathrm{by}$ keeping spherical surfaces only, the image quality is very bad and the spectrometer cannot be used.

This spectrometer is optimized in a first time by changing the spherical surfaces to fourth- and sixth- order aspherical surfaces. After optimization, the spectrometer presents a better image quality over [500 nm, $1100 \mathrm{~nm}]$ and the volume of the spectrometer is reduced to $100 \mathrm{~cm}^{3}$. Finally, the spectrometer is optimized for freeform surfaces. The all-freeform Offner spectrometer comprises only freeform surfaces. The performance is diffraction limited over the full specified FOV and the full specified spectral domain for a volume still equal to $100 \mathrm{~cm}^{3}$ Figure 4 . By keeping the same size, the design can be optimized to work at [200 nm, $1900 \mathrm{~nm}$ ] with $10 \mathrm{~mm}$ slit or at [200 nm, $1500 \mathrm{~nm}$ ] with $20 \mathrm{~mm}$ slit. 

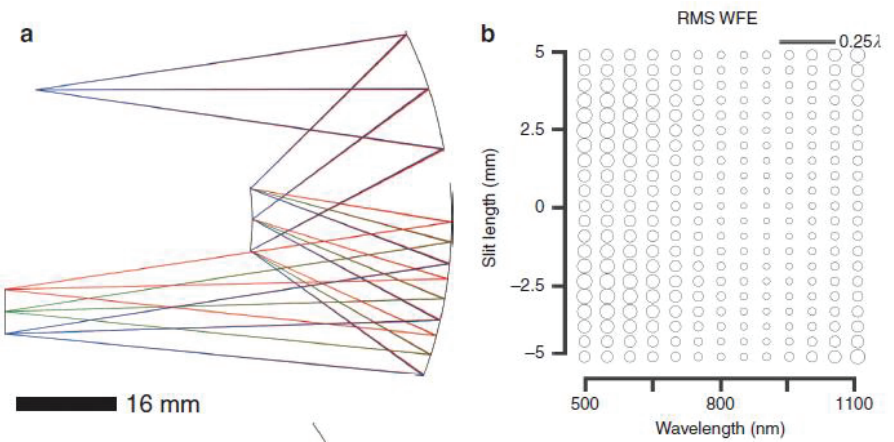

Figure 4. Freeform Offner spectrometer optical design layout with the wavefront error map over 0.5-1.1 $\mu \mathrm{m}$ spectral domain.

\subsection{Conical diffraction Offner hyperspectral imaging spectrometer (CDO-HIS)}

The paper [9] describes an Offner hyperspectral imaging spectrometer designed to observe the global ocean. This instrument includes one convex blazed grating to produce a "nearly non distortion and high spectral fidelity image". In particular, the Offner configuration has been selected to get a compact solution with high performance and a low distortion in wide spectral band.

The CDO-HIS comprises a push-broom imaging covering [400 nm, $900 \mathrm{~nm}$ ]. The F-number is equal to 4 and the FOV is equal to 0.9 degree that is very close to the FOV of the instrument we plan to design in the framework of the CNES study. The slit width (respectively length) is equal to $0.1 \mathrm{~mm}$ (respectively $1 \mathrm{~mm}$ ). The dispersion width is specified to be equal to $10.4 \mathrm{~mm}$ and the spectral resolution is specified to be better than $5 \mathrm{~nm}$. The Keystone and Smile are specified to be lower than 0.1 pixel.

The design is based on an all-spherical Offner configuration as shown in Figure 5. The mirrors are spherical and the diffraction grating is conical. The authors mention that this grating was fabricated by holographic lithography/ion-beametching techniques. The grating blaze angle is equal to $6.4^{\circ}$. The grating radius of curvature is around $50 \mathrm{~mm}$ with diameter of $16 \mathrm{~mm}$. The grating groove shape was optimized to improve the $1 \mathrm{st}$ order diffraction efficiency and the broadband response. The calculation had been done through a finite element analysis of the groove profile. The grating efficiency has been measured and is higher than 0.5 over the full specified spectral band. One filter is installed in front of the detector to minimize the straylight due to the spectral order overlapping.

The authors state that the theoretical system is almost diffraction limited with a very low level of distortion (less than 0.025 pixel).

The spectral resolution, smile distortion and key stone distortion have been measured at three different FOVs and at different spectral lines over the specified spectral domain. The average linear dispersion is $0.0206 \mathrm{~mm} / \mathrm{nm}$. The lowest spectral resolution is equal to $4.76 \mathrm{~nm}$. The smile distortion is equal to $0.28 \mathrm{~nm}$ or $5.6 \%$ of the pixel size. The keystone distortion is equal to $0.18 \mathrm{~nm}$ or $3.6 \%$ of the pixel size. 


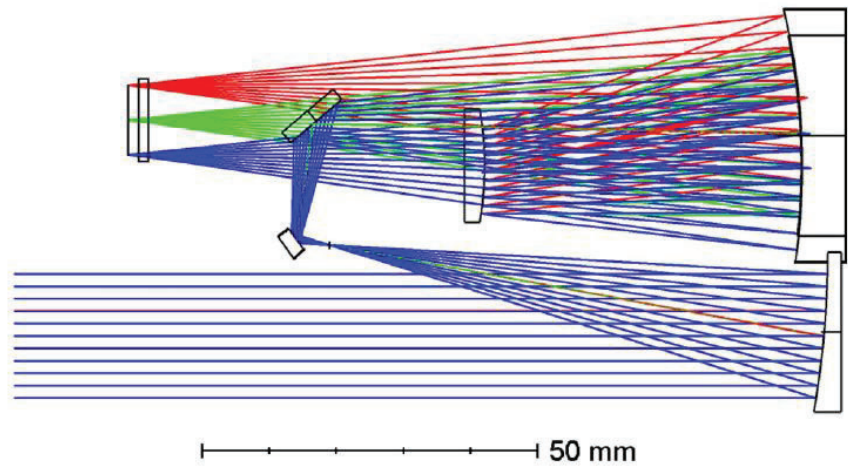

Figure 5. CDO-HIS spectrometer optical design layout.

\section{TMA SPECTROMETER}

\subsection{Double-pass freeform TMA}

The paper [17], which is the reference document mentioned by CNES, presents a comparison of 15 different systems: one Offner design, two Schwarzschild designs, two Dyson designs and ten TMA designs. The specified spectral domain is equal to $420-1000 \mathrm{~nm}$. The spectral dispersion is equal to $6.5 \mathrm{~nm} /$ pixel. The aperture is equal to $\mathrm{f} / 3$. The distortion is accessed by using two criteria: the smile and the keystone. The distortion is specified to be less than $20 \%$ of the pixel size, equal to $24 \mu \mathrm{m}$. The authors conclude that the following systems present better performance: the double-pass TMA, the Schwarzschild design and the Offner design.

We propose to focus on the double pass TMA that presents the advantage of including a plane diffraction grating as represented in Figure 6. The mirrors M1 and M3 are aspherical surfaces that could be manufactured on the same substrate to simplify the assembly process and relax the tolerances. The mirror M2 is the eighth-order freeform surface described by generalized Forbes polynomials. The authors propose to place one filter in front of the detector to eliminate the unwanted light from the diffraction orders. The system volume is equal to $120 \times 190 \times 240 \mathrm{~mm}^{3}$. The theoretical image quality is diffraction limited and the keystone is less than $5 \%$ and the smile is less than $12 \%$ of the pixel size.

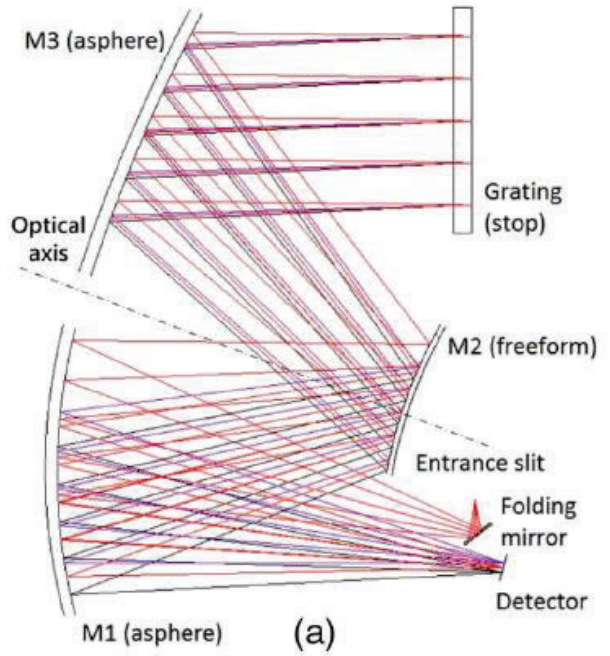

Figure 6. Double-pass TMA optical design layout. 


\section{DYSON SPECTROMETER}

\subsection{Compact Wide Swath Imaging Spectrometer (CWIS)}

This paper [22] presents the compact wide swath imaging spectrometer (CWIS). The instrument is a push-broom imaging spectrometer operating over the spectral domain $[380 \mathrm{~nm}, 2510 \mathrm{~nm}]$. The swath is equal to 52 degree, the slit length is equal to $48 \mathrm{~mm}$ that covers 1600 pixels in the FOV direction. The aperture number is equal to $\mathrm{F} \#=1.8$ and the specified spatial resolution is comprised between 0.3 and $20 \mathrm{~m}$ depending on the spectral band. The specified signal to noise ratio is higher than 2000 at wavelength equal to $450 \mathrm{~nm}$.

This spectrometer is a Dyson design with a volume equal to $325 \times 150 \times 150 \mathrm{~mm}^{3}$ and a mass less than $7 \mathrm{~kg}$. The CWIS spectrometer includes one achromatic doublet made of $\mathrm{CaF} 2$ and Fused-silica glasses to provide acceptable performance over the full specified FOV and spectral domain. The optical design layout is presented in Figure 7.

The most critical component of the spectrometer is the convex diffraction grating. The profile of the grating groove has been designed to provide a maximum grating efficiency over the full specified spectral domain. The groove profile differs significantly from the classical groove shape and requested specific manufacturing methods.

This concave grating was fabricated by the Jet Propulsion Laboratory, using electron beam lithography technique and diamond turning, then post polishing to minimize stray light. The grating diameter is equal to $125 \mathrm{~mm}$ and the sag is greater than $6 \mathrm{~mm}$. The theoretical efficiency is close to 0.62 at $\lambda=1750 \mathrm{~nm}$ and drops to about 0.49 at the wavelength $\lambda=2500 \mathrm{~nm}$. The efficiency is lower than 0.3 at the wavelength range below $1200 \mathrm{~nm}$.

The image quality performance is compatible with a pixel size of $30 \mu \mathrm{m}$. The smile and keystone distortions are about $1 \%$ of the pixel size.
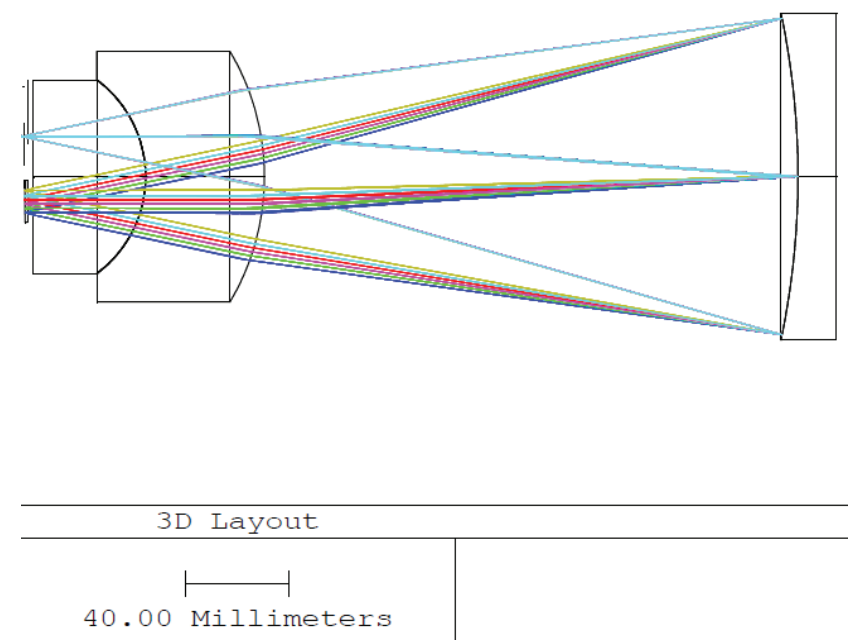

Figure 7. CWIS spectrometer optical design layout.

\subsection{LWIR Dyson}

In the paper [19], the authors describe one Dyson spectro imager operating in the LWIR spectral domain, from $7.8 \mu \mathrm{m}$ to $13.4 \mu \mathrm{m}$. It is important to mention that this spectral domain is different from the VNIR and SWIR spectral domains specified for our study. However, we consider that the LWIR Dyson spectrometer proposed in this paper identifies interesting options to design a compact Dyson spectrograph that could be used to design a similar instrument operating in the VNIR and SWIR spectral bands.

The F-number is equal to 2.5. The Dyson lens and aspherical lens are made of Zinc Selenide. The layout of the modified Dyson spectrometer is shown in Figure 8. The slit and the focal plane are located at a given distance close to the plane surface of the Dyson lens. This, in order to make sure that the slit and the Focal Plane Array can be accommodated. The air distances between the Dyson lens and the slit or the focal plane introduce some spherical aberration that degrades the performance. 
The authors propose two solutions to correct the spherical aberration: introducing an aspherical grating into the design or an aspherical lens in front of the spherical concave grating. The design presented in the paper includes a ZnSe aspherical lens located in front of the grating as represented in Figure 8.

The spot image over the specified spectral band and over the full slit is well contained in one pixel of $40 \mu \mathrm{m}$. The smile and keystone are less than $2 \mu \mathrm{m}$. The volume of the spectrometer is equal to $130 \times 50 \times 50 \mathrm{~mm}^{3}$, making this solution particularly interesting for our study.

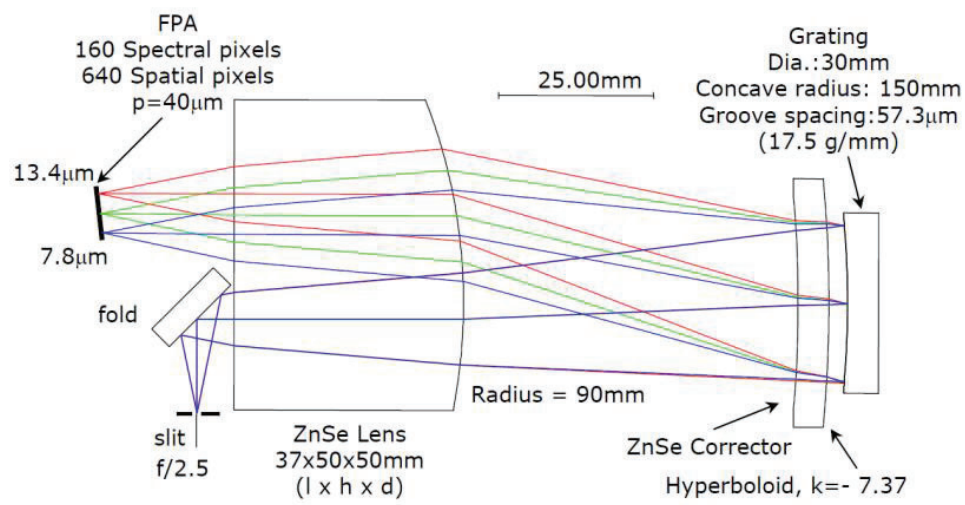

Figure 8. LWIR Dyson spectrometer optical design layout.

\section{GRATING DEVELOPMENT}

\subsection{Concave dual-period and variable-period gratings}

In the paper [23], the authors present the performance of gratings manufactured at Durham University's Precision Optics Laboratory by using a five-axis diamond-turning machine. This machine can manufacture i) multi-blaze structure gratings to maximize the diffraction efficiency over a larger spectral range and ii) variable spaced grooves to consider and compensate the projection effect of the grating's lines on a curved surface. The other advantages are the possibility to manufacture large metallic objects in substrates (such as nickel) thermally stable and compatible with cryogenic operations.

There are also two main limitations. The first limitation is the wearing of the diamond tip that could affect the homogeneity of the groove profile on the large surface thus impacting the grating efficiency. The second limitation is the thermal variation that could occurred during long time machining and that could induce surface errors.

The diameter of the grating is equal to $40 \mathrm{~mm}$. The grating groove density is equal to 100 line/mm. The grating is optimized to work at $1 \mathrm{st}$ diffraction order on the visible band [400 nm, $700 \mathrm{~nm}$ ]. The resolving power is equal to 4500 . The spectrometer F-number is equal to 6.6.

Four freeform grating are considered in this study. The gratings are presented in Figure 9. The Grating 1 is made of Aluminum RSA 6061 with constant line frequency optimized for $\lambda=588 \mathrm{~nm}$. The Grating 2 is made of Aluminum RSA 443 and plated with NiP with a constant line frequency optimized for $\lambda=588 \mathrm{~nm}$. The Grating 3 is made of RSA 443 and $\mathrm{NiP}$ with dual blazed structure optimized for $\lambda=470 \mathrm{~nm}$ and $\lambda=1047 \mathrm{~nm}$. The Grating 4 is made of RSA43 and NiP with a variable period optimized for $\lambda=588 \mathrm{~nm}$. The maximum differentiation of the variable period is equal to $20 \mathrm{~nm}$.

The NiP was identified as the substrate offering the lowest roughness and the best profile quality. The NiP offers an average roughness of $2.5 \mathrm{~nm}$ RMS per facet. The typical groove profiles measured shows that the profile machined on NiP is sharper than RSA6061. 


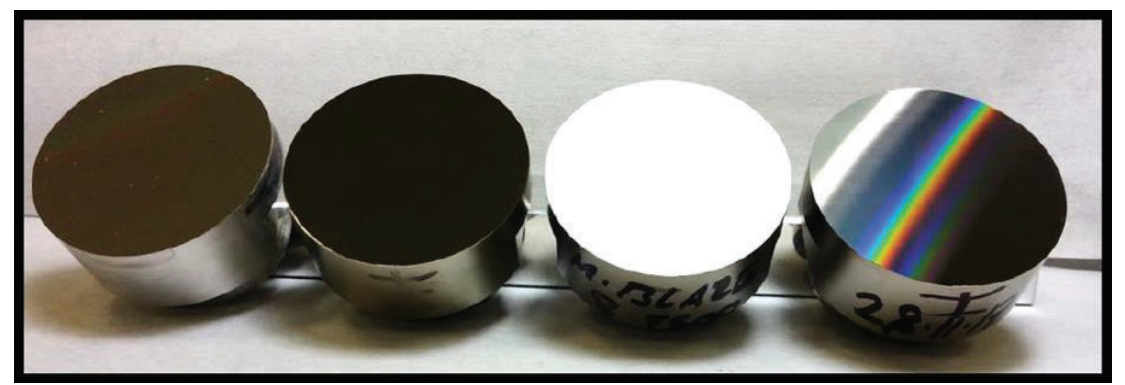

Figure 9. Freeform diffraction gratings manufactured using a 5-axis CNC machine.

\subsection{Convex blaze grating}

This paper [25] present a specific kind of spectrometer based on an Offner configuration and that includes a multi-slit system located in an intermediate plane. The BATMAN spectrometer was developed for 3.6m Telescopio Nazionale Galileo ground base telescope. The "Smart slit" is programmable to select the section on the FOV that will be sent to the spectrometer. The spectral domain covers $400-800 \mathrm{~nm}$. The central wavelength is at $580 \mathrm{~nm}$. The spectrometer aperture number is equal to 4 . The volume of the spectrometer is equal to $1.4 \times 1.2 \times 0.75 \mathrm{~m}^{3}$ and the mass is equal to $400 \mathrm{~kg}$. The particularity of this instrument is that the multi-slit system is an active MOEMS component. This instrument has been designed for astronomical observations over the visible spectral band only. The proposed instrument is thus outside the scope of our study.

This paper presents the development status and the performance of a convex blazed grating which is very interesting in the framework of the development of freeform compact spectrometers. The rectangular grating manufacturing realization method was firstly done on the flat substrate as shown in Figure 10. The Sol-Gel is used to replicate and transfer the shape of the original grating onto the substrate. To apply this method to the curve substrate is to use a flexible replica with an additional process of reactive ion etching as shown in Figure 11. The grating coating is protected silver from Optics BALZERS. The BATMAN has a grating size of $63.5 \mathrm{~mm}$, radius of curvature of $225 \mathrm{~mm}, 5.7$ degree of blaze angle and 300 line $/ \mathrm{mm}$.

(1)

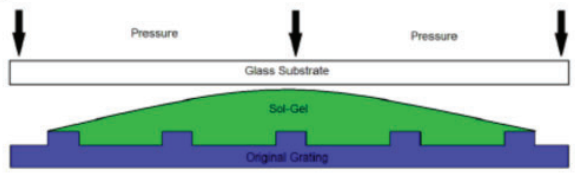

(3)

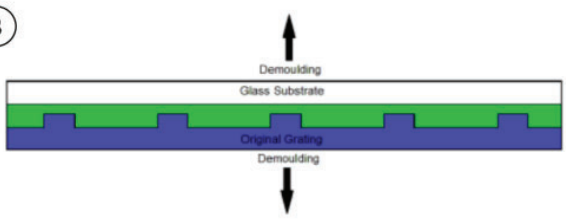

(2)

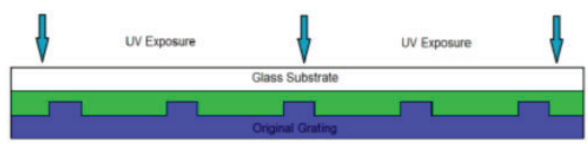

(4)

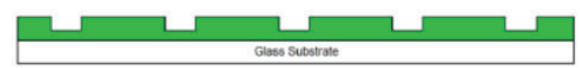

Figure 10. Grating replication process on a flat substrate. 

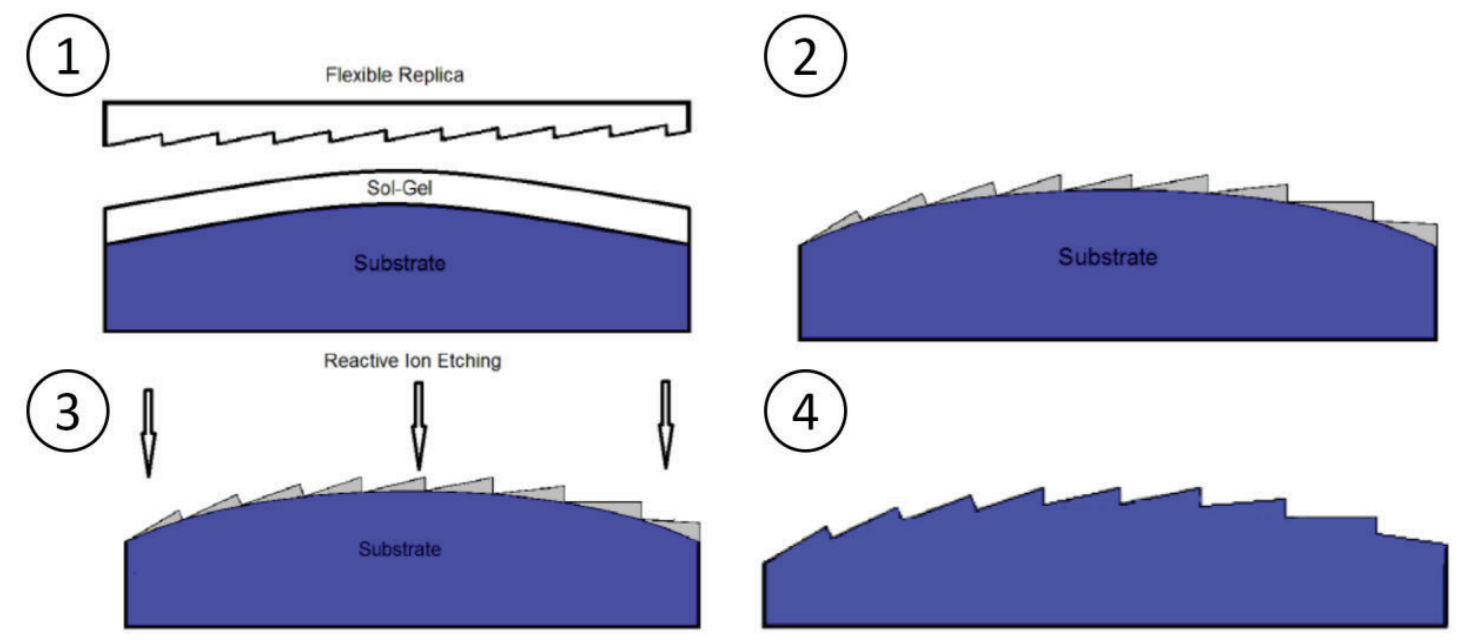

Figure 11. Grating replication process on a curve substrate.

\subsection{Surrey Satellite Technology (SSTL) spectrometer}

The SSTL spectrometer [27] is dedicated to monitoring atmosphere chemistry by using the absorption spectra of atmospheric gas species. The spectral resolution is equal to $0.5 \mathrm{~nm}$ in UV and VIS bands. The spectral resolution is equal to $0.1 \mathrm{~nm}$ in NIR and SWIR bands. The spatial resolution is equal to $300 \mathrm{~m}$ with $150 \mathrm{~km}$ swath width. The instrument includes silicon detectors for the UV, VIS and NIR bands and uses on HgCdTe detector for the SWIR band.

Two design of the spectrometer were proposed. The first spectrometer is specified for the Oxygen-A band $750-775 \mathrm{~nm}$ spectral interval (NIR band). The second spectrometer is specified for the SWIR band covering the spectral band of $2305-2385 \mathrm{~nm}$. The SWIR spectrograph comprises one blazed grating used in a quasi-Littrow configuration that provides an efficiency over $50 \%$.

The NIR spectrographs includes one immersed grating manufactured on the face of a prism as a dispersive element. The optical design layout is presented in Figure 12. The incident angle is comprised between 50 and 60 degree incidence angle in total internal reflection close to the Littrow condition that induces a high efficiency. It is worth to mention that collimator and the focusing lenses are identical in the proposed design.

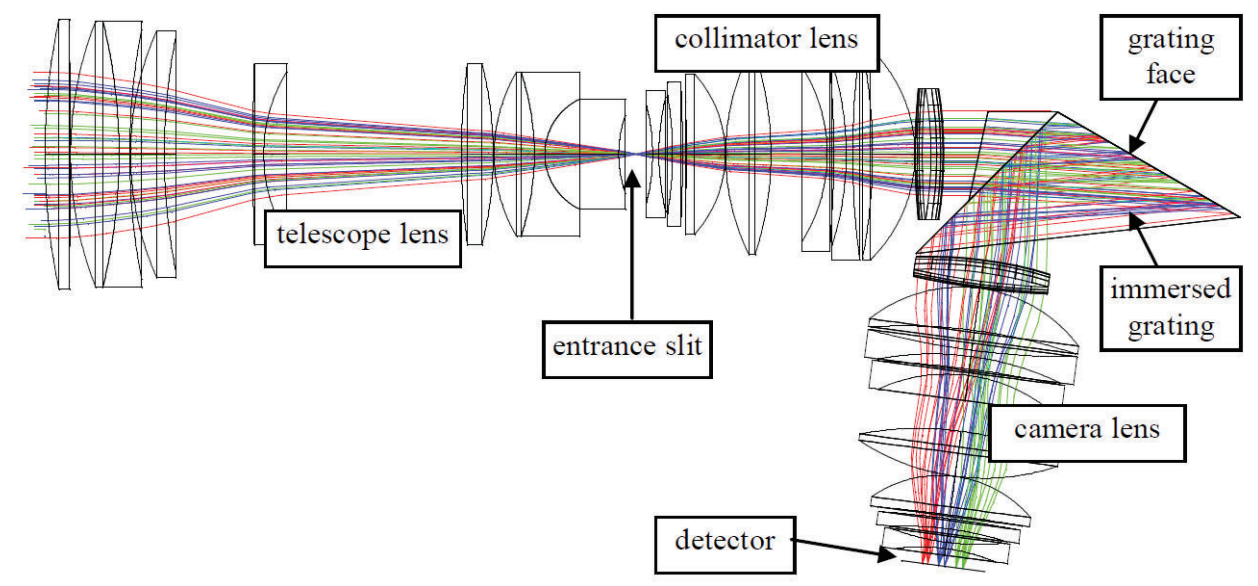

Figure 12. SSTL-NIR spectrometer layout 


\subsection{MicroCarb}

The objective of the CNES MicroCarb Satellite [28] flight mission is to monitor the carbon dioxide in Earth's atmosphere. The optical system has been designed to provide spectrum over four spectral bands: 764-768 nm, 1602-1619 $\mathrm{nm}$ and 2037-2065 nm for carbon dioxide and 1660-1672 nm for oxygen. One additional visible camera is installed before the spectrograph to image the observed scene.

The payload mass is $60 \mathrm{~kg}$. The volume is $1200 \times 700 \times 450 \mathrm{~mm}^{3}$. The telescope and the spectrometer are all made of freeform optics. The design is presented in Figure 13. There are three freeform mirrors for the telescope and three freeform mirrors for the spectrometer. These optics have been fabricated by Safran Reosc, with a surface size that varies between 50 and $200 \mathrm{~mm}$. The amplitude of the freeform surfaces is comprised between 100 and $1000 \mu \mathrm{m} \mathrm{PTV.} \mathrm{After}$ manufacturing, the surface roughness is equal to $2 \mathrm{~nm}$ and the WFE is better than $20 \mathrm{~nm}$ RMS.

The entrance sub-pupils are implemented to limit the field of each spectral band. In this optical system, the pupil separation prism (PSP) separates the entrance pupil into sub-pupil. Each sub-pupil will provide one beam for each spectral band. The field mask is implemented to eliminate the light from other sub-pupils.

The beam from the telescope is incident on the entrance slit and the Pupil Alignment Prisms (PAP). The PAP aims to recentering the entrance sub-pupil for each spectral band. The beam incident on the folding mirror FM1 is reflected toward the M3sp, M2sp and M1sp respectively. The M1sp reflects the beam toward the Echelle grating in near-Littrow configuration. The Echelle grating disperses the beam toward the M1sp, M2sp and M3sp respectively. The M3sp reflects the light toward the folding mirror FM2 and the detectors.

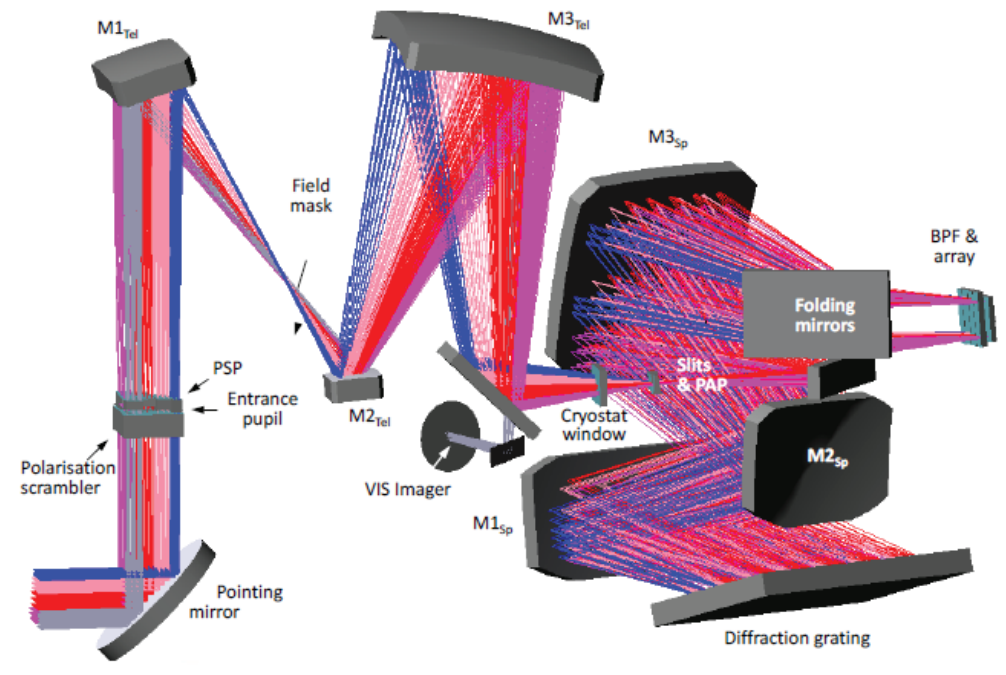

Figure 13 MicroCarb spectrometer layout

\section{CONCLUSIONS}

In this report, we presented the results of the review of thirty papers with the objective to identify the most promising compact spectrometer designs. We described the main characteristics of the state-of-art spectrometers optical design and we presented the technological status of freeform manufacturing capabilities. Based on the results of this survey, we decided to select three interesting systems: the Dyson optical design [22], the double-pass TMA [17] and the freeform Offner spectrometer [8].

First, we propose to study the Dyson instrument which represents the state-of-art compact spectrometer with performance in line with the need of our study. This optical configuration presents the advantage to provide good imaging performance, a wide swath and a fast aperture by using classical optical surfaces (no freeform optics).

Second, we selected the double pass freeform TMA that present the advantages to use a simple plane grating and two mirrors fabricated on a common substrate. Another advantage is that the double-pass TMA includes only one freeform surface thus minimizing the risk on the manufacturing capability when compared to a full-freeform surfaces 
spectrometer. The first drawback is that the double-pass configuration will require more stringent requirements on the optical surface quality and on the alignment accuracy. The second drawback is that the high number of optical surfaces will reduce the throughput of the instrument.

Finally, we propose to study the freeform Offner that has been identified as the most compact system proving the best image quality over a large spectral interval. However, this instrument involves three freeform surfaces, including the grating. This full freeform instrument will thus induce high manufacturing cost and a complex alignment procedure. Furthermore, the manufacturability of the grating on the freeform surface must be verified.

Table 1. Trade-off and volume of freeform Offner, double-pass freeform TMA and CWIS Dyson spectrometers.

\begin{tabular}{|c|c|c|c|}
\hline Design & Advantage & Drawback & Volume $\left(\mathrm{mm}^{3}\right)$ \\
\hline $\begin{array}{l}\text { Freeform } \\
\text { Offner }\end{array}$ & $\begin{array}{l}\text { Very compact system and excellent } \\
\text { image quality over wide spectral } \\
\text { domain } \rightarrow \text { Best ratio performance } \\
\text { versus volume identified in the } \\
\text { literature }\end{array}$ & $\begin{array}{l}\text { Three freeform surfaces } \rightarrow \text { High } \\
\text { cost, difficult to align and } \\
\text { manufacture } \\
\text { Freeform surface grating } \rightarrow \text { Difficult } \\
\text { to manufacture, align and test }\end{array}$ & $70 \times 50 \times 30$ \\
\hline $\begin{array}{l}\text { Double-pass } \\
\text { freeform TMA }\end{array}$ & $\begin{array}{l}\text { Plane grating is used in the design } \\
\rightarrow \text { Minimum risk and cost, eases the } \\
\text { alignment } \\
\text { M1 and M3 located on one common } \\
\text { substrate } \rightarrow \text { Simplify the assembly } \\
\text { and alignment process, relax the } \\
\text { tolerances, minimized the risk and } \\
\text { the cost } \\
\text { Only one freeform surface } \rightarrow \\
\text { Reduce the risk on the manufacturing } \\
\text { capability and eases the optical } \\
\text { alignment }\end{array}$ & $\begin{array}{l}\text { Incident beam of light reflected by } \\
\text { seven optical surfaces } \rightarrow \text { Tighten the } \\
\text { requirements on surface quality and } \\
\text { stability } \\
\rightarrow \text { Reduce the throughput of the } \\
\text { instrument }\end{array}$ & $120 \times 190 \times 240$ \\
\hline CWIS & $\begin{array}{l}\text { Wide swath and fast aperture are } \\
\text { provided } \rightarrow \text { Recent compact system } \\
\text { with specifications in line with the } \\
\text { needs of the study } \\
\text { No freeform surface } \rightarrow \text { Minimum } \\
\text { risk and cost, eases the alignment }\end{array}$ & $\begin{array}{l}\text { Big and thick lenses are used. } \\
\text { CaF2 material } \rightarrow \text { Fragile and } \\
\text { sensitive to the thermal variations } \\
\text { Detector and Slit located close to the } \\
\text { lens } \rightarrow \text { Increase the system } \\
\text { complexity } \\
\text { Grating } \rightarrow \text { special profile to manage } \\
\text { the very large spectral band }\end{array}$ & $325 \times 150 \times 150$ \\
\hline
\end{tabular}

\section{ACKNOWLEFGEMENT}

This work is carried out at the Astronomical Research Institute of Thailand (public organization) in the collaboration with Institut d'Optique Graduate School and Laboratoire Huber Curien. Additional financial support for research and survey was provided by Centre National d'Etudes Spatiales (CNES). W.W. would like to express his gratitude to Development and Promotion of Science and Technology Talents Project (DPST) for the PhD scholarship at Chiang Mai University. 


\section{REFERENCES}

[1] Terrence S. Lomheim, Eric A. Nussbaumer, Jeffrey A. Lang, David W. Warren, and Nemesio Caraballo "Parametric methodologies and tools for first-order hyperspectral imaging sensor system design", Proc. SPIE 5546, Imaging Spectrometry X, (15 October 2004)

[2] Fritz Blechinger, Bernd Harnisch, and Bernd Paul Kunkel "Optical concepts for high-resolution imaging spectrometers", Proc. SPIE 2480, Imaging Spectrometry, (12 June 1995)

[3] Thomas U. Kampe, Nathan Leisso, Robert Slusher, Paul Kaptchen, Nathan Showalter, Jerold Cole, Jonathan R. Fox, Kyle Solander, Lyle Ruppert, Rusty Schweickart, William S. Good, James Howell, Robert Warden, Frank Grochocki, James Baer, Laura Coyle, James Lasnik, Betsy Farris, and Emily Mrkvicka "The compact hyperspectral prism spectrometer for sustainable land imaging: enhancing capabilities for land remote sensing", Proc. SPIE 11127, Earth Observing Systems XXIV, 111270W (9 September 2019)

[4] Thomas U. Kampe, William S. Good, "Pathway to future sustainable land imaging: the compact hyperspectral prism spectrometer," Proc. SPIE 10402, Earth Observing Systems XXII, 1040208 (5 September 2017)

[5] Shanbing Zhu, Minxue Tang, Yiqun Ji, Guangbiao Gong, Ruirui Zhang, and Weimin Shen "Optical design of prism-grating-prism imaging spectrometers", Proc. SPIE 7156, 2008 International Conference on Optical Instruments and Technology: Optical Systems and Optoelectronic Instruments, 71560L (26 January 2009)

[6] Yong-hong Qi, Bin Xue, Yi-yi Zhao, and Jin-you Tao "Design of camera and spectrometer dual-use system", Proc. SPIE 8910, International Symposium on Photoelectronic Detection and Imaging 2013: Imaging Spectrometer Technologies and Applications, 891014 (30 August 2013)

[7] Lei Feng, Lidong Wei, Yunfeng Nie, Min Huang, Lei Yang, Xilu Fu, and Jinsong Zhou, "Design of a compact spectrometer with large field of view based on freeform surface", ELSEVIER 2019

[8] Reimers, J., Bauer, A., Thompson, K. et al., "Freeform spectrometer enabling increased compactness", Light Sci Appl 6, e17026 (2017)

[9] Qiao Pan, Xinhua Chen, Jiankang Zhou, Quan Liu, Zhicheng Zhao, and Weimin Shen, "Manufacture of the compact conical diffraction Offner hyperspectral imaging spectrometer," Appl. Opt. 58, 7298-7304 (2019)

[10] Yiqun Ji and Weimin Shen "Compact hyperspectral imaging system with a convex grating", Proc. SPIE 6834, Optical Design and Testing III, 68340Y (28 November 2007)

[11] Guangbiao Gong, Yiquan Ji, Shanbing Zhu, Ruirui Zhang, and Weimin Shen "Design of an off-axis threemirror system with high speed for hyperspectral imagers", Proc. SPIE 7156, 2008 International Conference on Optical Instruments and Technology: Optical Systems and Optoelectronic Instruments, 71561M (27 January 2009)

[12] Quan Liu, Yiqun Ji, Jianhong $\mathrm{Wu}$, and Weimin Shen "Study on convex grating in hyperspectral imaging spectrometers", Proc. SPIE 7494, MIPPR 2009: Multispectral Image Acquisition and Processing, 74940N (30 October 2009)

[13] Thomas Peschel, Matthias Beier, Christoph Damm, Johannes Hartung, Robert Jende, Sandra Müller, Mathias Rohde, Andreas Gebhardt, Stefan Risse, Ingo Walter, Ilse Sebastian, and David Krutz "Integration and testing of an imaging spectrometer for earth observation", Proc. SPIE 11180, International Conference on Space Optics - ICSO 2018, 1118000 (12 July 2019)

[14] T. Peschel, C. Damm, M. Beier, A. Gebhardt, S. Risse, I. Walter, I. Sebastian, and D. Krutz "Design of an imaging spectrometer for earth observation using freeform mirrors", Proc. SPIE 10562, International Conference on Space Optics - ICSO 2016, 1056237 (25 September 2017)

[15] A. Zuccaro Marchi, L. Maresi, and M. Taccola "Technologies and designs for small optical missions", Proc. SPIE 11180, International Conference on Space Optics - ICSO 2018, $111801 Z$ (12 July 2019)

[16] Stefan Kaiser, Bernhard Sang, Josef Schubert, Stefan Hofer, Timo Stuffler, "Compact prism spectrometer of pushbroom type for hyperspectral imaging," Proc. SPIE 7100, Optical Design and Engineering III, 710014 (27 September 2008)

[17] Chang Liu, Christoph Straif, Thomas Flügel-Paul, Uwe D. Zeitner, and Herbert Gross, "Comparison of hyperspectral imaging spectrometer designs and the improvement of system performance with freeform surfaces," Appl. Opt. 56, 6894-6901 (2017)

[18] John F. Silny and Thomas G. Chrien "Large format imaging spectrometers for future hyperspectral Landsat mission", Proc. SPIE 8158, Imaging Spectrometry XVI, 815803 (6 September 2011) 
[19] David W. Warren, David J. Gutierrez, Jeffrey L. Hall, and Eric R. Keim "Dyson spectrometers for infrared earth remote sensing", Proc. SPIE 7082, Infrared Spaceborne Remote Sensing and Instrumentation XVI, 70820R (3 September 2008)

[20] Robert Lucke and John Fisher "The Schmidt-Dyson: a fast space-borne wide-field hyperspectral imager", Proc. SPIE 7812, Imaging Spectrometry XV, 78120M (13 August 2010)

[21]B. Van Gorp, P. Mouroulis, D. W. Wilson, and R. O. Green "Design of the Compact Wide Swath Imaging Spectrometer (CWIS)", Proc. SPIE 9222, Imaging Spectrometry XIX, 92220C (15 September 2014)

[22] B. Van Gorp, P. Mouroulis, D. W. Wilson, R. O. Green, J. I. Rodriguez, E. Liggett, and D. R. Thompson "Compact Wide swath Imaging Spectrometer (CWIS): alignment and laboratory calibration", Proc. SPIE 9976, Imaging Spectrometry XXI, 997605 (19 September 2016)

[23] Cyril Bourgenot, Ariadna Calcines, and Ray Sharples "New opportunities of freeform gratings using diamond machining ", Proc. SPIE 10706, Advances in Optical and Mechanical Technologies for Telescopes and Instrumentation III, 1070626 (11 July 2018)

[24] Ariadna Calcines, Cyril Bourgenot, and Ray Sharples "Design of freeform diffraction gratings: performance, limitations and potential applications", Proc. SPIE 10706, Advances in Optical and Mechanical Technologies for Telescopes and Instrumentation III, $107064 Z$ (10 July 2018)

[25] Frédéric Zamkotsian, Igor Zhurminsky, Patrick Lanzoni, Nicolas Tchoubaklian, Christian Schneider, Sören Fricke, Marc Schnieper, Fabian Lütolf, Clément Luitot, and Vincent Costes "Convex blazed gratings for high throughput spectrographs in space missions", Proc. SPIE 11180, International Conference on Space Optics ICSO 2018, 1118051 (12 July 2019)

[26] Andrew Court, Jean Loup Bezy, Pieternel Levelt, Martin Siegl, Anton Leemhuis, Nick vd Valk, and Pepijn Veefkind "Compact hyperspectral instrument for NO2 remote sensing", Proc. SPIE 10785, Sensors, Systems, and Next-Generation Satellites XXII, 107850F (25 September 2018)

[27]D. R. Lobb and I. S. Bhatti "Applications of immersed diffraction gratings in Earth observation from space", Proc. SPIE 10565, International Conference on Space Optics - ICSO 2010, 105651M (20 November 2017)

[28] Frederick Pasternak, Philippe Bernard, Laurent Georges, and Véronique Pascal "The microcarb instrument", Proc. SPIE 10562, International Conference on Space Optics — ICSO 2016, 105621P (25 September 2017)

[29] Mark Herring, Thomas G. Chrien, Valerie G. Duval, and Timothy N. Krabach "Imaging spectrometry: concepts and system trade-offs", Proc. SPIE 1874, Infrared and Millimeter-Wave Engineering, (15 July 1993)

[30] Ajay Kumar, K., Nitesh Thapa and Kuriakose, S.A., "Advances in spaceborne hyperspectral imaging systems", Current Science, Vol. 108, No. 5, 10 March 2015 